\title{
Thermodynamics of Energy Systems and Processes: A Review and Perspectives
}

\author{
M. Feidt ${ }^{\dagger}$ \\ Professeur, Université Henri Poincaré, LEMTA - ENSEM \\ 2, avenue de la Forêt de Haye, Vandoeuvre-les-Nancy, 54516, France
}

†Corresponding Author Email: michel.feidt@ensem.inpl-nancy.fr

(Received July 16, 2010; accepted March 13, 2011)

\begin{abstract}
Thermodynamics is a relatively recent physical science that was born with calorimetry and thermometry experiments: so heat remains the central concept in relation with other forms of energy. The coupling between various forms is essential and related to conversion processes. The first conversion process that was analyzed was the thermomechanical one, at the time of Carnot. Equilibrium Thermodynamics was fruitful in connection with the efficiency concept, to qualify engines. But since that time, mass and heat transfers studies have been strongly developed (thermokinetics), as well as second law aspects of thermodynamics. It results new appraisal for energy systems and processes, relevant of a true thermodynamics approach. This was initiated by Onsager at the beginning of the 20th century, by analyzing the relation between fluxes and forces (gradients) from a general, but linear point of view. More recently, it was developed through a lumped analysis for systems by Chambadal and Novikov in 1957. It was rediscovered in 1975, by Curzon and Ahlborn. And since this work, a lot of books and publications have been proposed in the literature. A review of them is proposed here, on the basis of a synthesis due to the lack of place. The author's works are analysed and compared to the literature too. It results some original remarks and proposal relative to the obtained results: Comparison of entropy ratio method to entropy flux method, Comparison of endoreversible case to irreversible case, Comparison of adiabatic and non adiabatic systems, Comparison of constrained and non constrained systems. Main consequences of these comparisons are given, and future perspectives evoked on the main systems categories (engines; reverse machines; other eventual configurations).Conclusion is that FDOT (Finite Dimensions Thermodynamics) appears as a promising tool to be enlarged in the future.
\end{abstract}

Keywords: Energy systems and processes, Thermodynamics, Optimization

\section{NOMENCLATURE}

$\begin{array}{ll}\mathrm{A} & \text { area } \\ \dot{c} & \text { heat rate } \\ \mathrm{COP} & \text { coefficient of performance } \\ \mathrm{k} & \text { heat transfer conductance } \\ \dot{q} & \text { heat flux } \\ \dot{S} & \text { entropy flux } \\ \mathrm{T} & \text { temperature } \\ \dot{w} & \text { mechanical power } \\ w^{\prime} & \text { auxiliaries power } \\ \mathrm{X} & \text { temperature difference } \\ \mathrm{RM} & \text { Réfrigerating } \\ & \text { Machine } \\ \mathrm{S} & \text { source or sink } \\ & \text { system }\end{array}$

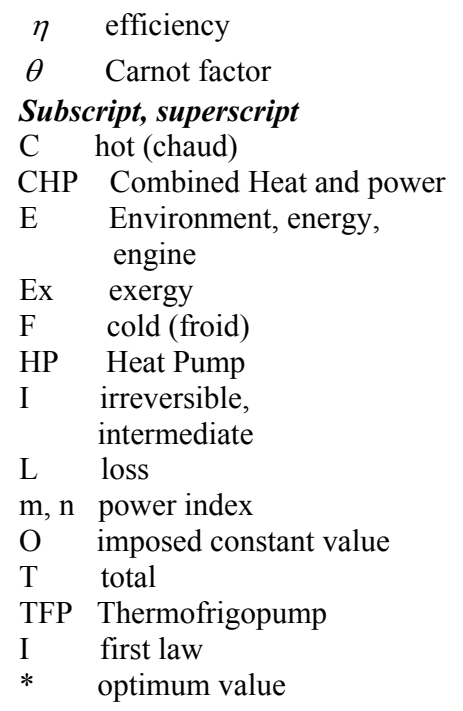




\section{INTRODUCTION}

Historically, Carnot (1824) was the first to develop a thermodynamical model for an engine, with an engineering point of view; but he considered too fundamental aspects: he his perhaps the father of the second law of thermodynamics, and its applications to engine and systems. He is also probably the inventor of the fruitful concept of efficiency. But the Carnot efficiency remains attached to equilibrium thermodynamics.

This limitation occurs until what was said the foundation paper of F.T.T. (Finite Time Thermodynamics) (Curzon and Ahlborn, 1975). Here are introduced the finite durations of Carnot engine thermodynamical processes: it results a finite mechanical power, and the corresponding efficiency appears less than the one corresponding to Carnot value.

This was first proposed in a slightly different form by Chambadal (1957) and simultaneously Novikov (1957). Today, this proposal is developed on a more accomplished form (Feidt 1996; Feidt 2006). Since the eighties, approximately twenty books have been published (see the specific annex), and numerous papers as well; the great majority of them are referenced in Bejan (1997), in Chen et al. (1999) and more recently in Durmayaz (2004).

The goal of the present paper is to confirm the interest of the proposed approach, and to enlighten the actual state of the subject, showing how the present progress of the thermodynamics move to escape from the equilibrium thermodynamics in direction of irreversible thermodynamics applied to systems and processes.

The present paper will be focused on Carnot cycles: direct cycle (engine) or reverse cycle (refrigerating machine or heat pump). The corresponding results are a synthesis of preceding author's works, the main point being that, contrarily to the great majority of published paper on the subject, the internal irreversibility of the machines (engine; refrigerating machine or heat pump) is accounted for originally (Feidt 2007). Interesting consequences are explored.

Numerous works remains to do, in order to obtain more complete (complex) results useful for engineers, but for physicists too, and developing entropy analysis (Bejan 1997). Fundamental aspects are carefully analyzed.

\section{The Time Dimension}

Within the scope of F.T.T., it seems logical to see explicit influence (not of time directly) but of durations of thermodynamical transformations. In that case (the most developed using F.T.T.), the calculated fluxes are generally mean values corresponding to the whole duration over the studied cycle. However, in various paper or communications, the fluxes appear directly, without explicit time variables, even if the model refers to F.T.T.

It seems preferable, according to the present proposal to characterize the corresponding model according to the steady state hypothesis (generally nominal conditions; full power for example), to rename it F.D.T. (Finite Dimension Thermodynamics); in that case these dimensions correspond to the size of the system, or some quantities related to this size, as we will see in the paper. The most used dimension in thermomechanical systems is relative to heat transfer conductances, $\mathrm{K}$, but it appears too areas $\mathrm{A}$, volume $\mathrm{V}$, heat rates $c$, heat flux $\dot{q}$ or power $\dot{w}$ limitations as well as temperature $\mathrm{T}$.

The suggestion is to reserve the F.T.T. spelling for transient conditions, where it is mandatory to take account of the time dimension.

Nevertheless in any case we agree with the fact that the time arrow is fundamentally connected to irreversibility, even if the time definition and concept remains to examine carefully. The hypothesis of steady state model is used hereafter, and the finite dimension chose is the generalized heat transfer conductance associated with.

\section{General Model of A THERMOMECHANICAL MACHINE}

\subsection{Fundamental laws}

The model is general because it describe a machine in contact with one heat source that is at constant temperature $\mathrm{T}_{\mathrm{SC}}$ (hot thermostat), and one heat sink at constant temperature $\mathrm{T}_{\mathrm{SF}}$ (cold thermostat). The machine could be an engine (direct cycle), a refrigerating machine or a heat pump (reverse cycle). The model is an extension of the Carnot cycle (Fig. 1 relative to engine). The first law of thermodynamics applied to the machine implies:

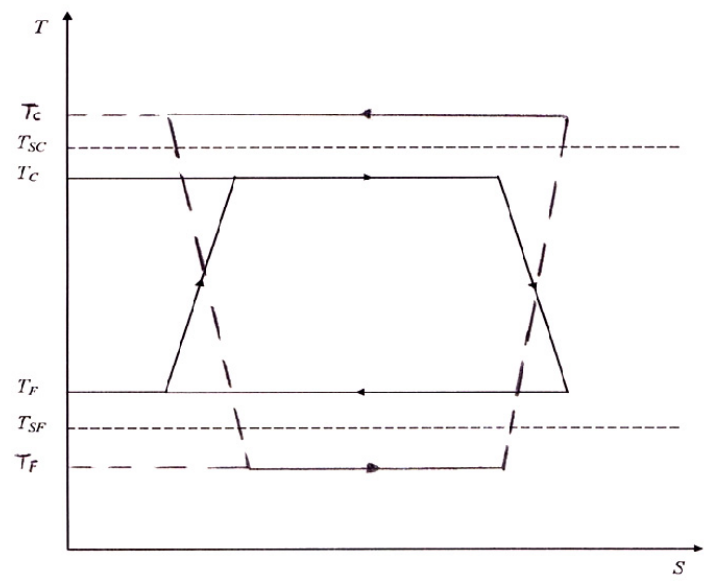

Fig. 1. Schematic of Carnot cycles with internal irreversibilities and external ones at contact to source and sink.

$q_{C}+\dot{q}_{F}=\dot{w}+w^{\prime}$

$q_{C}$, heat flux at the hot side of the machine ( $>0$, if recepted by the machine)

$q_{F}$, heat flux at the cold side of the machine 
$w$, mechanical power $(>0$, for an engine) produced or consumed

$w^{\prime}$, auxiliaries power (always $<0$, whatever is the machine)

The machine is connected to the heat source and heat sink through two heat transfer conductances respectively $\mathrm{K}_{\mathrm{C}}, \mathrm{K}_{\mathrm{F}}$.

Heat fluxes $q_{C}$ and $q_{F}$ are allowed through temperature differences respectively $\mathrm{X}_{\mathrm{C}}=\mathrm{T}_{\mathrm{SC}}-\mathrm{T}_{\mathrm{C}}, \mathrm{X}_{\mathrm{F}}$ $=\mathrm{T}_{\mathrm{SF}}-\mathrm{T}_{\mathrm{F}}$

The machine connected to heat source and sink, is the studied thermomechanical system.

If the system is non adiabatic, there are heat losses. The most convenient form to represent these losses is to suppose they occur between the maximum hot temperature $\mathrm{T}_{\mathrm{SC}}$ and the minimum low temperature $\mathrm{T}_{\mathrm{SF}}$, as a first approximation trough an equivalent global heat loss conductance $\mathrm{K}_{\mathrm{L}}$; it correspond the heat loss

flux $\dot{q}_{L}$ (choose as a positive quantity).

So the heat flux consumed at the hot source becomes:

$\dot{q}_{S C}=\dot{q}_{C}+\dot{q}_{L}$

The heat flux yielded at the cold sink becomes:

$q_{S F}=q_{F}-q_{L}$

The second law of thermodynamics applied to the machine gives the entropy balance:

$\frac{q_{C}}{T_{C}}+\frac{q_{F}}{T_{F}}+\dot{S}_{i}=0$

$s_{i}$, entropy flux created inside the machine, due to internal irreversibilities. As a first approximation we

suppose $S_{i}$ is a constant value parameter (for demonstrating purpose). This parameter is easily accessible through entropy analysis, as will be precised hereafter.

The second law of thermodynamics applied to the system gives:

$\frac{q_{S C}}{T_{S C}}+\frac{q_{S F}}{T_{S F}}+\dot{S}_{T}=0$

\subsection{Heat Transfer laws}

To complete the model the heat transfer laws are to be given; the general definition supposed is:

$\dot{q}=K . f\left(T_{S}, T\right)$

$\mathrm{K}$, generalized heat transfer conductance, $\mathrm{T}_{\mathrm{S}}$, external temperature relative to source of sink, $\mathrm{T}$, internal temperature relative to cycled fluid in the machine.

The heat transfer laws generally suppose the function $\mathrm{f}$ depending on $\mathrm{X}=\mathrm{T}_{\mathrm{S}}-\mathrm{T}$, such that:
$q=K . f(X)$

In the literature, the most used law are:

- $\quad$ the convective law $\dot{q}=K X^{n}$

with some particular case ( $\mathrm{n}=1,25$, Dulong Petit law ; $\mathrm{n}=1$ Newton law ; etc.) ; Newton law corresponds to linear approximation (Onsager, 1931)

- the radiative law $\dot{q}=K\left(T_{S}{ }^{m}-T^{m}\right)$

with some particular case $(\mathrm{m}=4$, Stefan law $; \mathrm{n}=-1$; phenomenological law, etc.) ; if $\mathrm{m}=1$, the radiative law corresponds too to linear approximation.

Some publications give results corresponding to all these heat transfer laws. Here we limit the purpose to linear approximation: $\dot{q}=K\left(T_{S}-T\right)=K . X$

\subsection{Finite Dimensions (Non Dimensionality)}

The system is always limited in size for physical and also cost reasons; so it implies here to introduce a limitation for heat transfer conductance:

$K_{C}+K_{F}=K_{T}$

$\mathrm{K}_{\mathrm{T}}$, total heat transfer conductances to be allocated appears as a size reference for the system.

The system too is immersed in an environment characterized by the ambient temperature $\mathrm{T}_{\mathrm{SE}}$ (but not only the temperature). This temperature appears as the reference temperature for the thermomechanical system.

It is to be noted that:

- for the great majority of engines, $\mathrm{T}_{\mathrm{SF}}=\mathrm{T}_{\mathrm{SE}}$ (excepted for combined heat and power engine where $\mathrm{T}_{\mathrm{SF}}>\mathrm{T}_{\mathrm{SE}}$ for example)

- for refrigerating machines generally, $T_{\mathrm{SC}}=\mathrm{T}_{\mathrm{SE}}$ (excepted for cascade refrigerating machine)

- for heat pump, $\mathrm{T}_{\mathrm{SF}}=\mathrm{T}_{\mathrm{SE}}$ (excepted for cascade heat pump)

- for reverse machine with two useful effects (at hot and cold side : thermofrigopump), we have $\mathrm{T}_{\mathrm{SF}}<\mathrm{T}_{\mathrm{SE}}<\mathrm{T}_{\mathrm{SC}}$

We propose to nondimensionnalize all the model equations with reference to $\mathrm{K}_{\mathrm{T}}$ and $\mathrm{T}_{\mathrm{SE}}$. It comes:

$k=\frac{K}{K_{T}} ; t=\frac{T}{T_{S E}} ; q=\frac{q}{K_{T} T_{S E}} ;$

$w=\frac{\dot{w}}{K_{T} T_{S E}}, x=t_{s}-t$ and $s=\frac{\dot{s}}{K_{T}}$

$q_{C}+q_{F}=w+w^{\prime}$

$\frac{q_{C}}{t_{C}}+\frac{q_{F}}{t_{F}}+s_{i}=0$

$\frac{q_{C}}{t_{S C}}+\frac{q_{F}}{t_{S F}}+S_{t}=0$

$k_{C}+k_{F}=1$ 
Remark: if $\mathrm{K}_{\mathrm{T}}$ is considered as a quantity to minimize (design or cost objective), it is necessary to choose as reference a unit heat transfer conductance, in order to retrieve $\mathrm{k}_{\mathrm{T}}$.

\subsection{Conclusion of Modeling}

The four equations of the model are completed by the two heat transfer laws:

$q_{C}=k_{C} \cdot f_{C}\left(x_{C}\right)$

$q_{F}=k_{F} \cdot f_{F}\left(x_{F}\right)$

It appears four main variables $\left(\mathrm{t}_{\mathrm{C}}, \mathrm{t}_{\mathrm{F}}, \mathrm{k}_{\mathrm{C}}, \mathrm{k}_{\mathrm{F}}\right)$, four intermediate variables $\left(\mathrm{q}_{\mathrm{C}}, \mathrm{q}_{\mathrm{F}}, \mathrm{w}, \mathrm{s}_{\mathrm{T}}\right.$; eventually $\left.\mathrm{k}_{\mathrm{T}}\right)$, four parameters $\left(\mathrm{t}_{\mathrm{SC}}, \mathrm{t}_{\mathrm{SF}}, \mathrm{w}^{\prime}, \mathrm{s}_{\mathrm{i}}\right)$

The model has two degrees of freedom allowing optimization.

\section{Performance Criterions of MACHINE OR SYSTEM}

As noted in section 1, the concept of efficiency was first proposed by Carnot for engine. Afterwhat it was enlarged; we summarize and comment in the following subsection 4.1 the classical energy efficiencies of thermomechanical machines.

\subsection{Energy Efficiencies}

These efficiencies are defined according to the first law of thermodynamics; from a general point of view, it could be defined as the ratio of a Useful Effect, U.E. divided by Energy Consumed, E.C. (Feidt 2009 a, b, c).

According to the sign convention used here, this definition gives:

- for an engine $\mathrm{E}$ :

$\eta_{E E}=\frac{w+w^{\prime}}{q_{C}}=\frac{w-|w|}{q_{C}}$

It appears that the presence of auxiliaries necessary to move the engine decrease the engine efficiency and consequently has to be accounted for. The engine efficiency is always less than one.

- $\quad$ for a Refrigerating Machine R.M.:

$C O P_{R M}=\frac{-q_{F}}{w+w^{\prime}}$

- $\quad$ for a Heat Pump

$C O P_{H P}=\frac{q_{C}}{w+w^{\prime}}$

Remark: in any case, we note that due to Eq. (7) $C O P_{H P}$ $=C O P_{R M}+1 ; C O P$ are generally greater than one, contrarily to $\eta$, engine efficiency.

To these three classical cases, we add two common interesting ones related to more than one useful effect; this extension corresponds to more integrated systems.

The first example is a Combined Heat and Power machine C.H.P.; the most common configuration is concerned with the water Vapour Turbine (T.V.). It comes:
$\eta_{C H P}=\frac{w+w^{\prime}-q_{F}}{q_{C}}=1$

This result indicates clearly the inadequacy of the first law approach in that case, due to the same "value" of all energies used (heat or mechanical energy); the T.V. is functioning at a temperature in between $\left[\mathrm{T}_{\mathrm{F}}, \mathrm{T}_{\mathrm{C}}\right]$ and consequently could not be adiabatic; consequently it appears internal losses of the vapor turbine to the environment $\mathrm{q}_{\mathrm{LTV}}$, and the efficiency is moved to:

$\eta_{C H P}=1-\frac{q_{L T V}}{q_{C}}$

It corresponds to an "adiabaticity" efficiency of the T.V.

The same reasoning as for CHP machine is applied to "Thermo Frigo Pump" T.F.P., where two simultaneous Useful Effects are used at the hot and the cold side of the machine; it comes the corresponding $\mathrm{COP}_{\mathrm{TFP}}$ :

$C O P_{T F P}=\frac{q_{C}-q_{F}}{w+w^{\prime}}=C O P_{H P}+C O P_{R M}$

We have to note here that the two useful effects correspond to heat fluxes delivered at different temperatures, respectively $\mathrm{T}_{\mathrm{C}}$ and $\mathrm{T}_{\mathrm{F}}$.

\subsection{Exergy Efficiency}

The concept of exergy was first proposed by Gouy (1889) and Stodola. This fruitful concept is a solution to overcome the lack of precision due to the first law of thermodynamics. It could be retrieve simply subtracting Eq. (8) from Eq. (7); we obtain this way the exergy balance of the machine :

$q_{C}\left(1-\frac{1}{t_{C}}\right)+q_{F}\left(1-\frac{1}{t_{F}}\right)-s_{i}=w+w^{\prime}$

In Eq. (18) appears the Carnot factor $\theta=\left(1-\frac{1}{t}\right)$ of every heat flux, and the corresponding thermal exergy, $q . \theta$.

According by subtracting Eq. (9) from Eq. (7), we obtain the exergy balance of the system:

$q_{C}\left(1-\frac{1}{t_{S C}}\right)+q_{F}\left(1-\frac{1}{t_{S F}}\right)-s_{T}=w+w^{\prime}$

This system exergy balance Eq. (19) is clearly different from the one of the machine Eq. (18) ; they coincide only in the limit of equilibrium thermodynamics $\left(t_{\mathrm{SC}}=\right.$ $\mathrm{t}_{\mathrm{C}} ; \mathrm{t}_{\mathrm{SF}}=\mathrm{t}_{\mathrm{F}}$ ).

Further, subtracting Eq. (18) from Eq. (19), we obtain:

$\mathrm{s}_{\mathrm{t}}=q_{C}\left(\frac{1}{t_{C}}-\frac{1}{t_{S C}}\right)+q_{F}\left(\frac{1}{t_{F}}-\frac{1}{t_{S F}}\right)+s_{i}$

So whatever is the kind of machine, the total entropy flux created is the sum of the entropy created at the external contact of the machine with source and sink (external irreversibilities for the machine), and the 
internal entropy created inside the machine, $s_{i}$. This approach confirms the difference between endo and exoirreversibilities relative to the studied machine. This method of distinction between endoirreversibility and exoirreversibility with respect to the thermomechanical machine could be easily generalized.

Consequently exergy efficiencies could be defined; but here, we have to differentiate between exergy efficiency of the machine, and exergy efficiency of the system. It appears that this difference is not so clear until now in the literature.

\subsubsection{Exergy Efficiencies of Machines}

The general definition of exergy efficiency is transposed from the energy one: it corresponds to the ratio of Useful Exergy U.Ex divided by Exergy Consumed Ex.C. It comes:

- for the engine :

$$
\eta_{E_{x} E}=\frac{w+w^{\prime}}{q_{C}\left(1-\frac{1}{t_{C}}\right)}=\frac{\eta_{E E}}{1-1 / t_{C}}
$$

- $\quad$ for the Refrigerating Machine :

$$
\eta_{E_{x} R M}=\frac{q_{F}\left(1-\frac{1}{t_{F}}\right)}{w+w^{\prime}}=-C O P_{R M}\left(1-\frac{1}{t_{F}}\right)
$$

- $\quad$ for the Heat Pump

$$
\eta_{E_{x} H P}=\frac{q_{C}\left(1-\frac{1}{t_{F}}\right)}{w+w^{\prime}}=C O P_{H P}\left(1-\frac{1}{t_{C}}\right)
$$

These results could be easily extended to C.H.P. machine and Thermo Frigo Pump. It comes:

- $\quad$ for the C.H.P. machine :

$$
\eta_{E_{x} C H P}=\frac{w+w^{\prime}-q_{F}\left(1-\frac{1}{t_{F}}\right)}{q_{C}\left(1-\frac{1}{t_{C}}\right)}=1-\frac{s_{i}}{q_{C}\left(1-\frac{1}{t_{C}}\right)}
$$

The exergy efficiency of a C.H.P. machine is less than one, due to internal irreversibility of the machine.

- $\quad$ for the T.F.P. machine :

$$
\eta_{E_{x} T F P}=\frac{q_{C}\left(1-\frac{1}{t_{C}}\right)+q_{F}\left(1-\frac{1}{t_{F}}\right)}{w+w^{\prime}}=1-\frac{s_{i}}{w+w^{\prime}}
$$

The exergy efficiency of a T.F.P. machine is also less than one, for the same reason as for the C.H.P. machine.

Consequently the exergy efficiency of the machine is representative of internal irreversibilities of the machine (endo efficiency of the machine or converter).

\subsubsection{Exergy Efficiencies of Systems}

The same demarche as developed in subsection 4.2.1. furnishes by analogy exergy efficiencies of machines connected to source and sink, $\eta_{E X S}$ :

- $\quad$ for the engine system

$$
\eta_{E x E S}=\frac{w+w^{\prime}}{q_{c}\left(1-\frac{1}{t_{S C}}\right)}=\frac{\eta_{E E}}{1-\frac{1}{t_{S C}}}
$$

- $\quad$ for the refrigerating system

$$
\eta_{E x R S}=\frac{q_{F}\left(1-\frac{1}{t_{S F}}\right)}{w+w^{\prime}}=-C O P_{R M}\left(1-\frac{1}{t_{S F}}\right)
$$

- $\quad$ for he heat pump system

$$
\eta_{E x H P S}=\frac{q_{C}\left(1-\frac{1}{t_{S C}}\right)}{w+w^{\prime}}=C O P_{H P}\left(1-\frac{1}{t_{S C}}\right)
$$

It is clear that these three system exergy efficiencies are dependent of Carnot factor relative to ambiance and respectively heat source $\left(t_{\mathrm{SC}}\right)$ and heat $\operatorname{sink}\left(\mathrm{t}_{\mathrm{SF}}\right)$.

They differ fundamentally from the one defined for the machines (subsection 4.2.1). They always are inferior to one.

- $\quad$ for the C.H.P. system it comes :

$$
\eta_{E x C H P S}=\frac{w+w^{\prime}-q_{F}\left(1-\frac{1}{t_{S F}}\right)}{q_{C}\left(1-\frac{1}{t_{S C}}\right)}=1-\frac{s_{T}}{q_{C}\left(1-\frac{1}{t_{S C}}\right)}
$$

fot he T.F.P. system :

$$
\eta_{E x T F P S}=\frac{q_{C}\left(1-\frac{1}{t_{S C}}\right)+q_{F}\left(1-\frac{1}{t_{S F}}\right)}{w+w^{\prime}}=1-\frac{s_{T}}{w+w^{\prime}}
$$

It appears that going from the machine efficiency (Eq. (24) or (25)) to the system efficiency (Eq. (29) or (30)) the exergy efficiency decreases, due to the adding of heat transfer irreversibilities (see Eq. (20)): internal and external irreversibilities occur in the system analysis.

Whatever in the case the machine exergy efficiency could be one only in the endoreversible case and the system exergy efficiency only in the endo and exo reversible case (this reversible case corresponds to equilibrium thermodynamics).

\section{OPTIMIZATION Without AND With ConstrainTs}

\subsection{Reconsideration of Objective Function: first law efficiency or Energy Efficiency}

As was seen in subsection 3.4 , the modeling of a thermomechanical machine exhibits physically two degrees of freedom; so optimization is theoretically possible. 
Without adding supplementary constraint, the present available literature shows that machine or system performance is mainly characterized by first law efficiency (subsection 4.1., Eqs. (13) to (17)). Due to the intercalation between $\mathrm{COP}_{\mathrm{HP}}$ and $\mathrm{COP}_{\mathrm{RM}}$, optimization of Eq. (14), (15), or (17) gives the same solution ; but as has been first considered previously (Blanchard, 1980, Goth, 1986) for reverse cycle machine a constraint must be added (finite energy) due to the fact that $t_{C}, t_{F}$ variables are not theorically bounded (see Fig. 1).

\section{Exergy efficiency}

We extent presently the performance analysis for machines and systems to exergy efficiencies (subsection 4.2.1). This approach is not fairly well considered today in the literature, even if it is developing fast: it corresponds to Eqs. (21) to (25) for machines, and Eqs. (26) to (30) for systems.

\section{Useful effect optimization}

This was the first attempt to escape from equilibrium Thermodynamics (Curzon and Ahlborn (1975); Chambadal (1957); Novikov (1957)): for an engine, the maximum power was sought for.

Afterwhat, were considered the refrigerating power of a refrigerating machine, and (or) the heating power of a heat pump.

These three cases are the basic ones; extensions are easy to other configurations as C.H.P. or T.F.P. machines.

From a general point of view, whatever is the machine, it corresponds to maximization of the Useful Effect U.E.

\section{Energy Consumption optimization}

This important case is practically ignored in the literature. We suggest that it could be an important objective function corresponding to efficient use of available energy; for a thermomechanical engine, it corresponds to minimization of $\mathrm{q}_{\mathrm{C}}$; for a refrigerating machine or heat pump to minimization of $\left|w+w^{\prime}\right|$.

\section{Ecological criterions (Angulo Brown, 1991)}

The environmental issue becomes more and more important today. Some specifically related to Thermodynamics are proposed in the literature. It seems that in any case these criterions are in connection with rejections $\mathrm{R}$.

For the thermomechanical machine presented, it corresponds to heat rejection (thermal pollution); so, it comes that the corresponding objective function consist to minimize the rejection $\left|q_{F}\right|$. The methodology is easy to generalize to any kind of rejection, and could be extended through L.C.A. Life Cycle Analysis.

Economical criterions (Bejan, 1996)

These criterions are numerous, and considered by engineers specifically;

\section{Investment cost}

$\mathrm{C}_{\mathrm{i}}$ could be related to the dimensions of the machine, through unitary cost $\mathrm{C}_{\mathrm{i}}$. For example, considering the physical dimension of the thermomechanical machine related to $\mathrm{K}$, the heat transfer conductances (section 2), minimization of investment cost suppose to know $\mathrm{C}_{\mathrm{ic}}$ the unitary cost of $\mathrm{K}_{\mathrm{C}}$ (hot side), respectively $\mathrm{C}_{\mathrm{iF}}$ of $\mathrm{K}_{\mathrm{F}}$ (cold side).

If the two costs are identical, it results a dimensional minimization of the machine in terms of $\mathrm{K}_{\mathrm{T}}$, with an optimal allocation of the $\mathrm{K}_{\mathrm{s}}$, too.

Operating cost $\mathrm{C}_{\mathrm{O}}$ : it is directly related to Energy Consumption, this consumption being characterized by an unitary $\operatorname{cost} \mathrm{c}_{\mathrm{O}}$ (for a fuel, $\mathrm{c}_{\mathrm{fo}}$ ); but it is to be noted, that it could appear some extra cost in relation with rejection (tax for example) ( $c_{o r}$, unitary cost for rejection)

\section{Global cost $\mathrm{Cg}$}

this cost to be minimized consists int the sum of all the costs. Some complementary economical criterions are also possible: the revenue (to maximize) or the benefit, and return time.

\section{Conclusion of the non constrained optimization}

It appears that possible objective functions are numerous, starting from efficiencies, to Useful Effects, Consumptions, Rejections, Economic criterions ; the last one refer to THERMOECONOMY essential for engineer, and environmental criterions.

Consequently, multiobjective optimization remains to develop fast.

We propose here to focus on physical (thermodynamical) simple objective function; taking account of the three main aspects enlighten, to say

\section{- U.E. Useful Effect \\ - C. Consumption (energy or exergy) \\ - R. Rejection}

These three ones are dimensional criterions. And we add the extended non dimensional ones related to Efficiency.

To these fundamental physical criterions, we suggest extending work of Bejan (1997), to add the two entropy related criterions : minimization of $s_{i}$, entropy created inside the machine, and (or) minimization of $\mathrm{s}_{\mathrm{T}}$, entropy created inside the system; these two entropy criterions corresponds to a minimization of energy dissipation inside machine (respectively system). Minimization of $\mathrm{s}_{\mathrm{i}}$ supposes to determine this quantity for the machine through entropy analysis. The same methodology is practicable for the system. However, minimization of $\mathrm{s}_{\mathrm{T}}$ is possible, even if $s_{i}$ is supposed a given constant parameters (to be experimentally identified). It is the way we choose in the following section for simplicity.

\subsection{Constrained Optimization}

The constrained optimization proposed uses the remark that frequently machines and systems are designed with a fixed objective. For example for a thermomechanical engine the power to delivered coal be imposed, or today appears some limitations in rejections ; in other case 
limitation could be due to the fuel availability, as in solar and wind energy systems. These are the three main possible constraints. But it is easy to define other ones like temperature limitations due to material $\left(\mathrm{T}_{\mathrm{MAX}}\right)$ for example, or limitation in pressure $\left(\mathrm{P}_{\mathrm{MAX}}\right)$.

We propose hereafter to examine the effect of the three main limitations on some objective functions to define as seen in subsection 5.1. Table 1 gives an overview of the set of cases to be examined; some of them are existing in the literature; others are completely new to our knowledge. Some cases are connected: for example, an engine with imposed power $w_{0}$, is such that the minimum of consumed heat flux $\dot{q}_{C}$ corresponds to maximum of energy efficiency $\eta_{E E}$ (this reduces the number of cases to study).

Table 1 synthesis of constrained engine optimization cases

\begin{tabular}{|c|c|c|c|c|c|c|c|}
\hline $\begin{array}{l}\text { Const } \\
\text { O.F. }\end{array}$ & Without & $w_{0}$ & $q_{C 0}$ & $\begin{array}{l}\eta_{0}< \\
\eta_{C}\end{array}$ & $S_{T}=S_{0}$ & $K_{T}=K_{0}$ & $\begin{array}{c}\text { Others... } \\
\text { (costs) }\end{array}$ \\
\hline$w$ & MAX & & MAX & MAX & MAX & MAX & \\
\hline$q_{c}$ & $\min$ & $\min$ & & $\min$ & $\min$ & $\min$ & \\
\hline$\eta$ & MAX & & & & MAX & MAX & \\
\hline$s_{T}$ & $\min$ & $\min$ & $\min$ & $\min$ & & $\min$ & \\
\hline$K_{T}$ & $\min$ & $\min$ & $\min$ & $\min$ & $\min$ & & \\
\hline $\begin{array}{l}\text { others } \\
\text { (costs) }\end{array}$ & & & & & & & \\
\hline
\end{tabular}

\subsection{Mathematical Method Used}

The method is based on variational calculus, and supposes to construct the lagrangian L of the study (lagrangian method); L is obtained knowing the objective function choose O.F., and all the constraints associated $\mathrm{C}_{\mathrm{i}}$ :

$L=F . O .+\sum_{i} \lambda_{i} C_{i}$

$\lambda_{i}$ is the lagrangian parameter.

\subsubsection{Optimization without Supplementary Constraint or With One}

These optimizations are respectively devoted to the following objective function

-for an engine $w, q_{C}, \eta, S_{T}$ with the constraints given by Eqs. (1), (2), (6) whatever is the heat transfer law.

-For a refrigerating machine (heat pump) $\dot{q}_{F}\left(\dot{q}_{C}\right), \dot{w}, C O P, \dot{S}_{T}$, with one constraint more than for the engine, as announced previously.

When $\mathrm{K}_{\mathrm{T}}$ is the objective function (design optimization), the last constraint Eq. (6) disappear as said previously (one degree of freedom more), and Eq. (1) gives the mechanical power associated to the studied case.
If we add one constraint more to the problem (see Table $1: w_{0}$, or $q_{C 0}$, or $\eta_{0}$, or $\left.C O P_{0}, \ldots.\right)$, we lose one degree of freedom, but optimization remains possible.

\subsubsection{Equations Systems to Solve}

They are obtained by derivation of the lagrangian with respect of variables $\left(t_{C}, t_{F}, k_{C}, k_{F}\right)$ and Lagrange

Parameters

The derivation with respect to Lagrange parameter gives back the constraint of the problem studied:

$$
\begin{aligned}
& \text { relation (1) }\left(\mathrm{C}_{1}\right): q_{C}+q_{F}-\left(w+w^{\prime}\right)=0 \\
& \text { relation (2) }\left(\mathrm{C}_{2}\right): \frac{q_{C}}{t_{C}}+\frac{q_{F}}{t_{F}}+s_{i}=0 \\
& \text { relation (6) }\left(\mathrm{C}_{3}\right): k_{C}+k_{F}=k_{T} \text { (or 1) } \\
& \text { relation (3) }\left(\mathrm{C}_{4}\right): \frac{q_{T}}{t_{S C}}+\frac{q_{F}}{t_{S F}}+s_{T}=0
\end{aligned}
$$

After elimination of Lagrange parameters, we get 4 equations with the four variables: the corresponding solution is the state vector at the optimum noted $\left(\mathrm{t}_{\mathrm{C}}\right.$ *, $\left.\mathrm{t}_{\mathrm{F}}{ }^{*}, \mathrm{k}_{\mathrm{C}}{ }^{*}, \mathrm{k}_{\mathrm{F}}{ }^{*}\right)$. Consequently the value of the objective function at the optimum O.F.* is determined, as well as any other useful value.

\section{EXamination of Some ObTained RESULTS}




\subsection{Tentative Synthesis of Results for the Engine}

General equations to solve, in order to get the optimum if it exists are summarized in Table 2. For the engine without supplementary constraints, same tables are available near the author for refrigerating machines and heat pumps. We use in this Table the following notations:

$q_{i, k}=\frac{\partial q_{i}}{\partial k_{i}} ; \quad q_{i, t}=\frac{\partial q_{i}}{\partial t_{i}} ; \quad\left(q_{i}\right)=\frac{q_{i, t} t_{i}-q_{i}}{q_{i, t} t_{i}{ }^{2}}$

The Table 3 corresponds to particular results of the engine, when the heat transfer law is the linear one. This Table confirms some existing results of the literature.

The maximum of efficiency for an endoreversible engine without thermal losses is the well known equilibrium thermodynamics limit.
The maximum of the engine power is associated with the well known CNCA (Chambadal - Novikov Curzon - Ahlborn) limit, generally spelled "nice radical" in the endoreversible limit. It seems other results are new formulated one, or at less unified ones.

Indeed we remark, that whatever is the treated case optimum heat transfer conductances are obtained trough $\left(\mathrm{C}_{2}\right)$ and $\left(\mathrm{C}_{3}\right)$ as:

$$
\begin{aligned}
& k_{C} *=\frac{k_{T}}{2}\left(1-\frac{s_{i}}{\alpha^{*}}\right) \\
& k_{F}^{*}=\frac{k_{T}}{2}\left(1+\frac{s_{i}}{\alpha^{*}}\right)
\end{aligned}
$$

Remark: however $\alpha^{*}$ is different, in each case (only formal identity when it exists). $\alpha$ is defined by: $\alpha=\frac{x_{C}}{t_{C}}=-\frac{x_{F}}{t_{F}}$.

\begin{tabular}{|c|c|}
\hline O.F. & Equations to solve at optimum \\
\hline$\eta_{I}$ & $\begin{array}{l}(\alpha): \frac{q_{C} \cdot q_{C, K}}{t_{C}{ }^{2} \cdot q_{C, t}}=\frac{q_{F} \cdot q_{F, K}}{t_{F}^{2} \cdot q_{F, t}} ; \quad q_{C}\left(q_{C}\right)+q_{F}=0 \\
\left(\mathrm{C}_{2}\right) ;\left(\mathrm{C}_{3}\right) \\
\text { remark : }\left(\mathrm{C}_{1}\right) \text { gives the }\left(w+w^{\prime}\right) \text { value associated to optimum }\end{array}$ \\
\hline$w+w^{\prime}$ & $\begin{array}{l}(\alpha): \frac{q_{C} \cdot q_{C, K}}{t_{C}{ }^{2} \cdot q_{C, t}}=\frac{q_{F} \cdot q_{F, K}}{t_{F}^{2} \cdot q_{F, t}} ; \quad\left(q_{C}\right)=\left(q_{F}\right) \\
\left(\mathrm{C}_{2}\right) ;\left(\mathrm{C}_{3}\right)\end{array}$ \\
\hline$q_{C}$ & $\begin{array}{l}(\alpha): \frac{q_{C} \cdot q_{C, K}}{t_{C}^{2} \cdot q_{C, t}}=\frac{q_{F} \cdot q_{F, K}}{t_{F}^{2} \cdot q_{F, t}} ; q_{F}, q_{C} \text { monotonous functions } \\
\left(\mathrm{C}_{2}\right) ;\left(\mathrm{C}_{3}\right): \text { one equation missing, one degree of freedom more } \\
\text { remark : }(\mathrm{C} 1) \text { gives the }\left(w+w^{\prime}\right) \text { value associated to optimum }\end{array}$ \\
\hline$s_{T}$ & $\begin{array}{l}(\alpha): \frac{q_{C} \cdot q_{C, K}}{t_{C}^{2} \cdot q_{C, t}}=\frac{q_{F} \cdot q_{F, K}}{t_{F}^{2} \cdot q_{F, t}} ; \quad t_{S C}\left(q_{C}\right)=t_{S F}\left(q_{F}\right) \\
\left(\mathrm{C}_{2}\right) ;\left(\mathrm{C}_{3}\right): \text { : one equation missing, one degree of freedom more } \\
\text { remark : }\left(\mathrm{C}_{1}\right) \text { gives the }\left(w+w^{\prime}\right) \text { value associated to optimum } \\
\qquad\left(\mathrm{C}_{4}\right) \text { gives the } s_{T} \text { value }\end{array}$ \\
\hline$k_{T}$ & $\begin{array}{l}(\alpha): \frac{q_{C} \cdot q_{C, K}}{t_{C}^{2} \cdot q_{C, t}}=\frac{q_{F} \cdot q_{F, K}}{t_{F}^{2} \cdot q_{F, t}} ; \quad\left(q_{C}\right)=\left(q_{F}\right) \\
\left(\mathrm{C}_{2}\right) \\
\text { remark : }\left(\mathrm{C}_{1}\right) \text { gives the }\left(w+w^{\prime}\right) \text { value } ;\left(\mathrm{C}_{4}\right) \text { gives the } s_{T} \text { value } \\
\text { one equation missing, one degree of freedom more }\end{array}$ \\
\hline
\end{tabular}

Table 2 Equations to determine the non constrained engine optimum values of variables 
Table 3 Analytical optimization results for an engine, with linear heat transfer laws, and without added constraint

\begin{tabular}{|c|c|}
\hline O.F. & Variables values at optimum \\
\hline$\eta_{I}$ & $\begin{array}{l}t_{C} *=\frac{t_{S C}}{1+\sqrt{s_{i}}} ; t_{F} *=\frac{t_{S F}}{1-\sqrt{s_{i}}} ; \eta_{I} *=1-\frac{t_{S F}}{t_{S C}}\left(\frac{1+\sqrt{s_{i}}}{1-\sqrt{s_{i}}}\right)^{2} \\
\left(w+w^{\prime}\right)^{*}=\frac{\sqrt{s_{i}}}{2}\left[t_{S C} \frac{1-\sqrt{s_{i}}}{1+\sqrt{s_{i}}}-t_{S F} \frac{1+\sqrt{s_{i}}}{1-\sqrt{s_{i}}}\right] ; q_{C}=\frac{1}{2} \sqrt{s_{i}} \frac{t_{S C}\left(1-\sqrt{s_{i}}\right)}{1+\sqrt{s_{i}}} \\
\text { remark : } \alpha^{*}=\sqrt{s_{i}} ; k_{C} *=\frac{k_{T}}{2}\left(1-\sqrt{s_{i}}\right)\end{array}$ \\
\hline $\begin{array}{c}w+ \\
w^{\prime}\end{array}$ & $\begin{array}{l}t_{C} *=\frac{\sqrt{t_{S C}}}{2}\left(\sqrt{t_{S C}}+\sqrt{t_{S F}}\right) ; t_{F} *=\frac{\sqrt{t_{S F}}}{2}\left(\sqrt{t_{S C}}+\sqrt{t_{S F}}\right) \\
\left(w+w^{\prime}\right)^{*}=\left(\frac{\sqrt{t_{C-}} \sqrt{t_{S F}}}{2}\right)^{2}\left[1-s_{i}\left(\frac{\sqrt{t_{S C}}+\sqrt{t_{S F}}}{\sqrt{t_{S C}}-\sqrt{t_{S F}}}\right)^{2}\right] \\
\eta_{I}=1-\sqrt{\frac{t_{S F}}{t_{S C}}} \cdot \frac{\sqrt{t_{S C}}-\sqrt{t_{S F}}+s_{i}\left(\sqrt{t_{S C}}+\sqrt{t_{S F}}\right)}{\sqrt{t_{S C}}-\sqrt{t_{S F}}-s_{i}\left(\sqrt{t_{S C}}+\sqrt{t_{S F}}\right)} ; \alpha * \frac{\sqrt{t_{S C}}-\sqrt{t_{S F}}}{\sqrt{t_{S C}}+\sqrt{t_{S F}}}\end{array}$ \\
\hline$q_{C}$ & $\begin{array}{l}q_{C}, \text { monotonous with } \alpha\left(q_{F} \text { also }\right): q_{C}=\frac{1}{2} t_{S C} \frac{\alpha-s_{i}}{\alpha+1} \\
\text { optimization in } k_{i} \text { only : } \\
k_{C} *=\frac{k_{T}}{2}\left(1-\frac{s_{i}}{\alpha}\right) ; k_{F} *=\frac{k_{T}}{2}\left(1+\frac{s_{i}}{\alpha}\right) \\
\text { remark : } \alpha \text { is parameter, or } t_{C}\left(x_{C}\right) \text {, or } t_{F}\left(x_{F}\right)\end{array}$ \\
\hline$s_{T}$ & $\begin{array}{l}s_{T}, \text { monotonous with } \alpha\left(\min s_{T}=\mathrm{s}_{\mathrm{i}}, \text { when } \alpha=0\right) \\
s_{T}=\frac{\alpha^{2}+s_{i}}{1-\alpha^{2}} \\
\text { optimization in } k_{i} \text { only : (see above) } \\
\text { remark : } \alpha \text { parameter, or } t_{C}\left(x_{C}\right), \text { or } t_{F}\left(x_{F}\right)\end{array}$ \\
\hline$k_{T}$ & $\begin{array}{l}\text { optimization in } t_{i}: t_{C} *=\frac{\sqrt{t_{S C}}}{2}\left(\sqrt{t_{S C}}+\sqrt{t_{S F}}\right) ; t_{F} *=\frac{\sqrt{t_{S C}}}{2}\left(\sqrt{t_{S C}}+\sqrt{t_{S F}}\right) \\
k_{T} \text {, monotonous with } k_{C} \text { or } k_{F} \text { (one degree of freedom) } ; k_{F} \text { has been choosed with limit }\left(k_{F}\right. \\
\left.\geq \frac{s_{i}}{2 \alpha^{*}}\right) ; k_{C}^{*}=k_{F}-\frac{s_{i}}{\alpha^{*}}, \alpha^{*}=\frac{\sqrt{t_{S C}}-\sqrt{t_{S F}}}{\sqrt{t_{S C}}+\sqrt{t_{S F}}} \\
\text { remark : } s_{T}=2 k_{F} \frac{\alpha^{* 2}}{1-\alpha^{* 2}}+\frac{s_{i}}{1-\alpha^{*}}\end{array}$ \\
\hline
\end{tabular}

Persons interested by extension to non adiabatic engines could refer to Feidt (2009), where there is also a comparison between the created entropy flux method, and the irreversibility ratio method. Results exist too for non adiabatic engines (Feidt, 2009). To illustrate the difference, we introduce the stagnation temperature of the system $\mathrm{T}_{\mathrm{S}}$ (maximum attainable temperature of the system is too temperature limitation), and using the proposed methodology optimizes the engine power with respect to the variables including $\mathrm{T}_{\mathrm{SH}}$ new system variable; it comes the corresponding results: 
Table 4 Equations and results for optimization of a Carnot engine with an added constraint and linear heat transfer laws

\begin{tabular}{|c|c|}
\hline $\begin{array}{c}\text { O.F. with } \\
\text { constraint }\end{array}$ & optimum of $\eta_{I}$ (a) \\
\hline$w+w^{\prime}=w_{0}$ & $\begin{array}{l}\alpha_{0}=\frac{\left(1+s_{i}\right)\left(t_{S C}-t_{S F}\right)-\sqrt{\Delta}}{2\left(t_{S C}+t_{S F}-2 w_{0}\right.} ; \Delta=\left(1+s_{i}\right)^{2}\left(t_{S C}-t_{S F}\right)^{2}- \\
-4\left(t_{S C}+t_{S F}-2 w_{0}\right)\left[s_{i}\left(t_{S C}+t_{S F}\right)+2 w_{0}\right] \\
k_{C} *=\frac{k_{T}}{2}\left(1-\frac{s_{i}}{\alpha_{0}}\right) ; k_{F} *=\frac{k_{T}}{2}\left(1+\frac{s_{i}}{\alpha_{0}}\right) \\
\quad q_{C}\left(\alpha_{0}\right)=\frac{1}{2} t_{S C} \cdot \frac{\alpha_{0}-s_{i}}{\alpha_{0}+1} ; \eta_{I}=w_{0} / q_{C}\left(\alpha_{0}\right) \\
\text { remark : } \Delta \text { introduces an inequality constraint on } s_{i}\end{array}$ \\
\hline$q_{C}=q_{C 0}$ & $\begin{array}{c}\alpha_{0}=\frac{s_{i} t_{S C}+2 q_{C 0}}{t_{S C}-2 q_{C 0}} ; \eta_{I} *=\frac{t_{S C}-t_{S F}}{t_{S C}} \cdot \frac{1-\frac{s_{i} t_{S C} t_{S F}+4 q_{C 0}{ }^{2}}{q_{C 0}\left(1-s_{i}\right)\left(t_{S C}-t_{S F}\right)}}{1-\frac{4 q_{C 0}}{1-s_{i} t_{S C}}} \\
\text { remark : } s_{T}=\frac{s_{i}+4\left(\frac{q_{C 0}}{t_{S C}}\right)^{2}}{1-s_{i}-4 \frac{q_{C 0}}{t_{S C}}} ; \text { monotonous with } s_{i}, q_{C 0}\end{array}$ \\
\hline O.F. with constraint & optimum of $\left(w+w^{\prime}\right)$ (b) \\
\hline$q_{C}=q_{C 0}$ & $\begin{array}{l}\left(\mathrm{w}+\mathrm{w}^{\prime}\right) *=\frac{q_{C 0}\left(1-s_{i}\right)\left(t_{S C}-t_{S F}\right)-s_{i} t_{S C} t_{S F}-4 q_{C 0}^{2}}{t_{S C}\left(1-s_{i}\right)-4 q_{C 0}} \\
\text { remark : solution formally identical to the above one }\end{array}$ \\
\hline$\eta_{I}=\eta_{0}$ & $\begin{array}{c}\alpha_{0}=\frac{\left(1+s_{i}\right)\left(\eta_{C}-\eta_{0}\right)-\sqrt{\Delta}}{2\left(2-\eta_{C}-\eta_{0}\right)} \approx \frac{s_{i}\left(2-\eta_{C}-\eta_{0}\right)}{\left(1+s_{i}\right)\left(\eta_{C} \eta_{0}\right)} \\
\text { avec } \Delta=\left(1+s_{i}\right)^{2}\left(\eta_{0}-\eta_{C}\right)^{2}-4 s_{i}\left(2-\eta_{C}-\eta_{0}\right)^{2} \geq 0 \\
\left(w+w^{\prime}\right)^{*}=\eta_{0} \frac{t_{S C}}{2} \frac{\alpha_{0}-s_{i}}{\alpha_{0}+1} \approx \eta_{0} \frac{t_{S C} s_{i}}{2} \cdot \frac{2\left(1-\eta_{C}\right)-s_{i}\left(\eta_{C}-\eta_{0}\right)}{\left(\eta_{C}-\eta_{0}\right)+2 s_{i}\left(1-\eta_{0}\right)}\end{array}$ \\
\hline $\begin{array}{l}\text { O.F. with } \\
\text { constraint }\end{array}$ & optimum of qC (c) \\
\hline$\eta_{I}=\eta_{0}$ & $\begin{array}{c}\alpha_{0}=\frac{\left(1+s_{i}\right)\left(\eta_{C}-\eta_{0}\right)-\sqrt{\Delta}}{2\left(2-\eta_{C}-\eta_{0}\right)} ; \Delta=\left(1+s_{i}\right)^{2}\left(\eta_{0}-\eta_{C}\right)^{2}-4 s_{i}\left(2-\eta_{C}-\eta 0\right)^{2} \\
\text { Formal identity to preceding case } \\
q_{C}^{*}=\frac{t_{S C}}{2} \cdot \frac{\left(\eta_{C}-\eta_{0}\right)-\sqrt{\Delta}-s_{i}\left(4-3 \eta_{C}-\eta_{0}\right)}{\left(4-3 \eta_{0}-\eta_{C}\right)-\sqrt{\Delta}+s_{i}\left(\eta_{C}-\eta_{0}\right)}\end{array}$ \\
\hline $\begin{array}{l}\mathrm{w}+\mathrm{w}^{\prime}= \\
=\mathrm{w} 0\end{array}$ & $\begin{array}{c}\alpha_{0}=\frac{\left(1+s_{i}\right)\left(t_{S C}-t_{S F}\right)-\sqrt{\Delta}}{2\left(t_{S C}+t_{S F}-2 w_{0}\right)} \\
\Delta=\left(1+s_{i}\right)^{2}\left(t_{S C}-t_{S F}\right)^{2}-4\left(t_{S C}+t_{S F}-w_{0}\right)\left[\left(t_{S C}+t_{S F}\right) s_{i}+2 w_{0}\right] \\
q_{C} *=\frac{1}{2} t_{S i} \frac{\alpha_{0}-s_{i}}{\alpha_{0}+1}\end{array}$ \\
\hline $\begin{array}{l}\text { O.F. with } \\
\text { constraint }\end{array}$ & optimum of sT (d) \\
\hline$\eta_{I}=\eta_{0}$ & $\begin{array}{c}\alpha_{0}=\frac{(a-1)\left(1+s_{i}\right)-\sqrt{\Delta}}{2(a+1)} ; \Delta=(a-1)^{2}-4 s_{i}(1+a)^{2} \geq 0 \\
\mathrm{a}=\frac{1-\eta_{0}}{1-\eta_{C}}>1\end{array}$ \\
\hline $\mathrm{w}^{+}+\mathrm{w}^{\prime}=\mathrm{w} 0$ & $\begin{array}{c}\text { Same solution as in table } 4 \text { (a) } \\
\text { Remark : if } \mathrm{qC}=\mathrm{qC0} \quad \text { (see table } 4 \text { (a) continuation) }\end{array}$ \\
\hline
\end{tabular}


$K_{C}{ }^{*}=\frac{K_{T} \sqrt{I}}{1+\sqrt{I}}$ (irreversibility ratio method)

or

$K_{c}^{*}=\frac{K_{T}}{2} \frac{1+\dot{s}_{i}\left(\frac{1}{K_{L}} \cdot \frac{\sqrt{T_{S F}}}{\sqrt{T_{S}}-\sqrt{T_{S F}}}+\frac{1}{K_{T}} \frac{\sqrt{T_{S}}+\sqrt{T_{S F}}}{\sqrt{T_{S}}-\sqrt{T_{S F}}}\right)}{1+\frac{s_{i}}{2 K_{L}} \cdot \frac{\sqrt{T_{S F}}}{\sqrt{T_{S}}-\sqrt{T_{S F}}}}$

And

$$
\eta_{E E S}(w *)=\frac{1}{1+\frac{K_{L}}{K_{T}}(1+\sqrt{I})^{2}} \cdot \frac{\left(\sqrt{T_{S}}-\sqrt{I T_{S F}}\right)^{2}}{T_{S}-T_{S F}}
$$

or

$$
\eta_{E E S}\left(w^{*}\right)=\frac{1}{1-\frac{\dot{S}_{i}}{K_{T}}+4 \frac{K_{L}}{K_{T}}}\left[\begin{array}{l}
\frac{\sqrt{T_{S}}-\sqrt{T_{S F}}}{\sqrt{T_{S}}+\sqrt{T_{S F}}}-\dot{S}_{i} \\
{\left[\frac{1}{K_{T}} \cdot \frac{\sqrt{T_{S}}-\sqrt{T_{S F}}}{\sqrt{T_{S}}-\sqrt{T_{S F}}}+\frac{1}{K_{L}} \frac{T_{S F}}{T_{S}-T_{S F}}\right]}
\end{array}\right]
$$

From these relations, it comes that internal irreversibilities are coupled to non adiabaticity. Constrained optimization results are also new ones. Some of them are reported hereafter for thermomechanical engines and linear heat transfer laws. Tables 4.a, 4.b, 4.c report respectively the constrained optimum of $\eta_{E E}$ corresponding to classical first law efficiency $\eta_{I}, w+w^{\prime}, q_{C}$ for the various indicated contraints.

In these cases the optimum heat transfer conductances allocation is always given by Eqs. (33) and (34) in which $\alpha^{*}$, the intermediate variable is becoming $\alpha$ (constrained intermediate variable : see the tables).

Table 4.d is relative to the objective function $\dot{S}_{T}$ to minimize in presence of supplementary constraint; it appears that only the case where the efficiency is imposed differs from the other ones.

Table 5 summarizes the results of what we said the "design" optimization of the engine $\left(\min \mathrm{K}_{\mathrm{T}}\right.$ ), always for linear heat transfer laws (particular analytical results).

Whatever is the supplementary constraint used, the design optimization of the engine corresponds to:

$\alpha^{*}=\frac{\sqrt{t_{S C}}-\sqrt{t_{S F}}}{\sqrt{t_{S C}}+\sqrt{t_{S F}}}=\frac{1-\sqrt{1-\eta_{C}}}{1+\sqrt{1-\eta_{C}}}$

with $\eta_{C}$, Carnot efficiency.

The heat conductance allocation remains in accordance with Eqs. (33) and (34).

\begin{tabular}{|c|c|}
\hline $\begin{array}{l}\text { O.F. with } \\
\text { constraint }\end{array}$ & Optimum of $K_{T}$ \\
\hline $\begin{array}{c}w+w^{\prime}= \\
\quad=w_{0}\end{array}$ & $\begin{array}{c}k_{T} * \frac{4 w_{0}+s_{i}\left(\sqrt{t_{S C}}+\sqrt{t_{S F}}\right)^{2}}{\left(\sqrt{t_{S C}}-\sqrt{t_{S F}}\right)^{2}} \\
\eta_{I}=\frac{w_{0}}{w_{0}+\frac{s_{i}}{2} \sqrt{t_{S F}}\left(\sqrt{t_{S C}}+\sqrt{t_{S F}}\right)}\left(1-\sqrt{\frac{t_{S C}}{t_{S F}}}\right) \\
s_{T}=\frac{1}{\left(\sqrt{t_{S C}}+\sqrt{t_{S F}}\right) \sqrt{t_{S C} t_{S F}}}\left[w_{0}\left(\sqrt{t_{S C}}-\sqrt{t_{S F}}\right)+\frac{s_{i} \sqrt{t_{S C}}}{2}\left(t_{S C}-t_{S F}+4 \sqrt{t_{S C} t_{S F}}\right)\right]\end{array}$ \\
\hline$q_{C}=q_{C 0}$ & $\begin{array}{c}k_{T} *=\frac{1}{\sqrt{t_{S C}}-\sqrt{t_{S F}}}\left[\frac{4 q_{0}}{\sqrt{t_{S C}}}+s_{i}\left(\sqrt{t_{S C}}-\sqrt{t_{S F}}\right)\right] \\
\eta_{I}=1-\sqrt{\frac{t_{S F}}{t_{S C}}}-\frac{s_{i}}{2 q_{0}} \sqrt{t_{S F}}\left(\sqrt{t_{S C}}-\sqrt{t_{S F}}\right) \\
s_{T}=\frac{\sqrt{t_{S C}}+\sqrt{t_{S F}}}{2 \sqrt{t_{S C}}}\left[\frac{2 q_{0}}{t_{S C}} \cdot \frac{\sqrt{t_{S C}}-\sqrt{t_{S F}}}{\sqrt{t_{S C}}+\sqrt{t_{S F}}}+s_{i}\right]\end{array}$ \\
\hline$\eta_{I}=\eta_{0}$ & $\begin{array}{c}k_{T} *=s_{i} \frac{\sqrt{t_{S C}}+\sqrt{t_{S F}}}{\sqrt{t_{S C}}-\sqrt{t_{S F}}}-\frac{1-\eta_{0}+\sqrt{t_{S F} / t_{S C}}}{1-\eta_{0}-\sqrt{t_{S F} / t_{S C}}} \\
q_{C}=\frac{s_{i} \sqrt{t_{S C}}}{2}\left(\sqrt{t_{S C}}+\sqrt{t_{S F}}\right) \frac{1}{\sqrt{t_{S C} / t_{S F} \cdot\left(1-\eta_{0}\right)-1}} \\
s_{T}=\frac{s_{i}}{2} \cdot \frac{\sqrt{t_{S C}}+\sqrt{t_{S F}}}{\sqrt{t_{S C}}} \cdot \frac{t_{S C} / t_{S F}\left(1-\eta_{0}\right)-1}{\sqrt{t_{S C} / t_{S F}\left(1-\eta_{0}\right)-1}}\end{array}$ \\
\hline
\end{tabular}

Table 5 Optimum design results $\left(\min \mathrm{K}_{\mathrm{T}}\right.$ ) for a Carnot engine with an added constraint and linear heat transfer laws 


\subsection{Extension to C.H.P and Series Connected Cycles}

\subsubsection{Combined Heat and Power Carnot System}

This extension of the modeling is straight forward (Feidt, 2009), but as indicated in section 4.2.2., the most significant objective function becomes the maximum useful exergy flux $\left|\dot{E} x_{u}\right|$; with heat reference relative to the useful fluid $\mathrm{T}_{\mathrm{u}}$ (for example vapour temperature) it comes :

Irreversibility for the ratio method :

$\left|\dot{E} x_{u}\right|=\dot{q}_{C}\left(1-I \frac{T_{0} T_{F}}{T_{u} T_{C}}\right)$

For the entropy flux method:

$\left|\dot{E} x_{u}\right|=\dot{q}_{C}\left(1-\frac{T_{0} T_{F}}{T_{u} T_{C}}\right)-\frac{T_{0} T_{F}}{T_{u}} \dot{s}$

Equation (39) is chosen. The corresponding optimum is:

$\operatorname{MAX}\left|E x_{u}\right|=\frac{K_{L}\left(\sqrt{T_{S}}-\sqrt{T_{0}}\right)\left[K_{T}\left(\sqrt{T_{S}}-\sqrt{T_{0}}\right)-\dot{s}_{i}\left(\sqrt{T_{S}}+\sqrt{T_{0}}\right)\right]}{K_{T}-\dot{S}_{i}+4 K_{L}}$

Supposing incoming energy flux is pure exergy, this corresponds to:

$\eta\left[M A X\left|\dot{E} x_{u}\right|\right]=\frac{1}{\sqrt{T_{S}}+\sqrt{T_{0}}} \cdot \frac{K_{T}\left(\sqrt{T_{S}}-\sqrt{T_{0}}\right)-\dot{S}_{i}\left(\sqrt{T_{S}}+\sqrt{T_{0}}\right)}{K_{T}-\dot{S}_{i}+4 K_{L}}$

As a partial conclusion, it can be noted that the entropy flux method permits a more precise identification of the influence of internal irreversibility and thermal losses.

Extension to the cases with an extra constraint is reported in (Feidt, 2009d).

\subsubsection{Series Connected Carnot Engines}

Modeling of a two Carnot cycles have been recently reported (Feidt, 2010), the objective function in that case being the total power of the engines "cascade", the

constraint being the available heat flux $\dot{q}_{C 0}$ at the $\mathrm{T}_{\mathrm{SC}}$ temperature. It comes easily in the endoreversible case for the O.F. first law efficiency, with linear heat transfer laws.

$$
\eta_{E_{\text {endo }}}=1-\frac{T_{S F}}{T_{S C}} \cdot \frac{1}{1-\frac{\dot{q}_{C 0}}{T_{S C}}\left[\frac{1}{K_{C}}+\frac{1}{K i}+\frac{1}{K_{F}}\right]}
$$

$\mathrm{K}_{\mathrm{i}}$, being the intermediate heat transfer conductance.

Introducing the finite dimension constraint as:

$K_{T}=K_{C}+K_{i}+K_{F}$

We recover the equipartition of conductances for the endoreversible system efficiency maximum:
$\eta_{E_{\text {endo }}}^{*}=1-\frac{T_{S F}}{T_{S C}} \frac{1}{1-9 \dot{q}_{0} / K_{T} T_{S C}}$

This interesting case corresponds in fact to O.R.C. systems (Organic Rankine Cycles). And it has been generalized to a N-O.R.C. cycles cascade, according to:

$\mathrm{K}_{\mathrm{i}}^{*}=\frac{K_{T}}{n+1}$

And the corresponding maximum efficiency

$$
\eta_{E_{\text {endo }}}^{*}=1-\frac{T_{S F}}{T_{S C}-\frac{\dot{q}_{C 0}(n+1)^{2}}{K_{T}}}
$$

\section{CONCLUSION}

- A review has been proposed here showing the evolution of machine and system modeling from Carnot appraisal (equilibrium Thermodynamics) to today tendencies represented by F.T.T. Finite Time Thermodynamics.

- We have shown how in fact great majority of studies are only implicitly related to time, but not explicitly due to steady state modeling essentially. It seems that F.T.T. spelling is more convenient for fully transient conditions machines or systems.

- The concept of efficiency of a machine and a system has been revisited. The necessary use of exergy efficiency has been proved for C.H.P. configurations, but also for thermofrigopump, two main illustrations. Also the difference between exergy efficiency of a machine and the corresponding system efficiency has been enlightened.

- Some comparison between entropy ratio method and entropy flux method, endoreversible and irreversible cases, adiabatic and non adiabatic configurations have been developed.

- Reconsideration of objective function has been done starting from classical efficiencies, through power of an engine, and new extensions proposed by the author recently (energy consumption; heat rejection).

- New results have been proposed for realistic cases where an added constrained is joined (mainly E.C. energy consumption, or U.E. useful effect, or imposed efficiency).

- A general methodology has been proposed for thermomecanical machines, with the associated set of equations to solve, whatever are the heat transfer laws.

- Main consequences of all the comparison are given and future perspectives too. Extension to reverse cycles machines and other combined configurations has been proposed; some results are available near of the author in this direction.

- Lastly we propose to call this approach F.D.T. Finite Dimensions Thermodynamics, where Dimensions means every physical limited quantity; this methodology appears a promising one to be enlarged in the future. 


\section{REFERENCES}

Angulo Brown, F. (1991). An ecological optimization criterion for finite time heat engines. J. Applied Physics 69(11), 7465-7469.

Bejan, A. (1997). Entropy generation minimization: the new thermodynamics of finite size, and finite time processes. Journal of Applied Physics 79, 11911218.

Bejan A., G. Tsatsaronis, M. Moran (1996). Thermal Design and optimization, J. Wiley and Sons Inc., New York, USA.

Blanchard, C.H. (1980). Coefficient of performance for finite speed heat pump. J. Appl. Phys. 51, 24712472.

Carnot, S. (2005). Réflexions sur la puissance motrice $d u$ feu sur les machines propres à developer cette puissance. Editions Jacques Gabay, Paris, France.

Chambadal, P. (1957). Les centrales nucléaires. A. Colin éditeur, Paris, France.

Chen, L., S. Wu, F. Sun (1999). Finite time thermodynamic optimization or entropy generation minimization of energy systems. Journal of Non Equilibrium Thermodynamics 24(4), 327-359.

Curzon, F.L., B. Ahlaborn (1975). Efficiency of a Carnot engine at maximum power output. American Journal for Physics, 22-24.

Durmayaz, A., O.S. Sogut, B. Sahin, H. Yavrez (2004). Optimization of thermal systems based on finite time thermodynamics and thermoeconomics. Prog. Energy Combust. Sci. 30(2), 175-217.

Feidt, M. (2009a). Thermodynamique et efficacité énergétique, invited lecture. Colloquium of the French Thermal Sociéty (SFT), Vannes, France.

Feidt, M. (2009b). Efficacité énergétique: quels critères. invited lecture CNT'09 Colloquium Brasov, Romania, Termotehnica 2, 25-32.

Feidt, M. (2009c). Energy efficiency and environment. accepted for publication in UPB Sci. Bull. Series.

Feidt, M. (2009d). Optimal thermodynamics - New upperbounds, Entropy 11, 529-547.

Feidt, M., M. Costea, C. Petre, S. Petrescu (2007). Optimization of the direct Carnot cycle. Applied Thermal Engineering 27(5-6), 829-839.

Feidt, M. (2006). Energétique, Concepts et applications. Dunod éditeur, Paris, France.

Feidt, M. (1996). Thermodynamique et optimisation énergétique des systèmes et procédés. TEC et DOC éditeur, Paris, France.
Goth, Y., and M. Feidt (1986). Recherche sur les conditions optimales de fonctionnement des pompes à chaleur ou machines à froid associées à un cycle de Carnot endoreversible. C.R. Acad. Sci. Paris 303(II)(1), 113-122.

Gouy, R. (1889). Sur l'énergie utilisable, Journal de Physique 8, 501.

Laheurte, G., M. Feidt, R. Boussehain, D. QueirosCondé, Cascade constructale de cycles moteurs, accepted for publication in ENSTA, Paris, France.

Novikov, I. (1957). The efficiency of atomic power stations (a review). Atomaya Energiya, 3-11.

Onsager, L. (1931). Physical Review 37, 405; Physical Review 38, 2265.

\section{Appendix \\ List of books B1 to B19}

B1 Chambadal P., Evolution et Applications du concept d'Entropie, Dunod éditeur, Paris, France, 1963.

B2 Bejan A., Entropy Generation through Heat and Fluid Flow, Wiley, New-York, 1982.

B3 Andresen B., Finite Time Thermodynamics, Physics Laboratory, University of Copenhagen, Denmark, 1983.

B4 Tsirlin A.M., Optimal Cycles and Cycle Regimes, Energomizdat, Moscow, USSR, (in russian), 1985.

B5 Feidt M., Thermodynamique et Optimisation énergétique des Systèmes et Procédés, TEC et DOC Lavoisier éditeur, PARIS, France, 1987.

B6 Bejan A., Advanced Engineering Thermodynamics, Wiley, New-York, 1988.

B7 Sieniutycz S., Salamon P., Advances in Thermodynamics: Finite Time Thermodynamics and Thermoeconomics, vol. 4, Taylon and Francis Editor, New-York, 1990.

B8 De Vos A., Endoreversible Thermodynamics of Solar Energy Conversion, Oxford University, Oxford, 1992.

B9 Bejan A., Entropy Generation Minimization, CRC Press, Boca Raton, 1996.

B10 Bejan A., Tsatsaronis G., Moran M.J., Thermal Design and Optimization, Wiley, NewYork, 1996.

B11 Bejan A., Feidt M., Moran M.J., Tsatsaronis G. , Proceeding ECOS'98 (two volumes), Nancy, France, 1998.

B12 Bejan A., Mamut E. , Thermodynamic Optimization of Complex Energy System, NATO 
M. Feidt / JAFM, Vol. 5, No. 2, pp. 85-98, 2012.

Science, Series 3, High Technology, Vol. 69, Kluwer Academic, 1999.

B13 Berry R.S., Kasakov V., Sieniutycz S., Szwast Z., Tsirlin A.M. , Thermodynamic optimization of Finite Time Processes, Wiley, New-York, 2000.

B14 Wu C., Chen L., Chen J., Recent Advances in Finite Time Thermodynamics, Nova Science Publishers, New-York, 2000.

B15 Sieniutycz S., De Vos A., Thermodynamics of Energy Conversion and Transport, Springer Verlag, 2000

B16 Gordon J.M., Ng K.C., Cool Thermodynamics; the Engineering and Physics of Predictive, Diagnostic and Optimization Methods for Cooling Systems, Cambridge International Science Publishing, U.K, 2000.

B17 Brodiansky V.M., Sorin M.V., Le Goff P. , The efficiency of industrial processes, exergy analysis and optimization, Energy Research 9, Elsevier, Amsterdam, 1994.

B18 Sciubba E., Melli R., Artificial intelligence in thermal systems design: concepts and applications, Nova Science Publishers, New-York, 1998.

B19 Radcenco V.T., Generalized Thermodynamics, Editura Tehnica, Bucharest, Romania, 1994. 